Article

\title{
Prediction of the Neutrino Mass Scale Using Coma Galaxy Cluster Data
}

\author{
Peter D. Morley
}

Blue Ridge Scientific LLC, P. O. Box 4871, Manassas, VA 20108, USA; peter3@uchicago.edu

Received: 6 June 2020; Accepted: 14 June 2020; Published: 23 June 2020

\begin{abstract}
The near degeneracy of the neutrino masses-a mass symmetry-allows condensed neutrino objects that may be the Dark Matter everybody is looking for. If the KATRIN terrestrial experiment has a neutrino mass signal, it will contradict the analysis of the Planck Satellite Consortium reduction of their raw cosmological microwave data. Using Condensed Neutrino Objects as the Dark Matter along with Coma Galaxy Cluster data, we predict that KATRIN will indeed see a neutrino mass signal. If this physics drama unfolds, there will be profound implications for cosmology, which are discussed in this paper.
\end{abstract}

Keywords: dark matter; condensed neutrino objects

\section{Introduction-What is at Stake Here}

The Planck Satellite Consortium (PSC) reduced their raw cosmological microwave data using the $\Lambda$ CDM model (lambda cold Dark Matter), with a critical assumption that neutrinos were in thermodynamic equilibrium with Baryonic Matter in the Early Universe. However, that assumption may be wrong [1]. If neutrino flavors are Dirac Fermions, they will possess a magnetic dipole moment $\vec{\mu}$. Radiation losses will ensue from the magnetic moment flipping about in Chaotic Early Universe magnetic fields [1]. Importantly, the radiation loss $P_{r a d}$ is independent of the tiny neutrino magnetic moment because it depends, instead, on its time-rate of change $|\dot{\vec{\mu}}|$, which varies from 0 to $\infty$

$$
P_{\text {rad }}=\frac{2}{3 c^{2}}\left[\gamma^{8}(\vec{\beta} \cdot \dot{\vec{\beta}})^{2} \dot{\vec{\mu}}^{2}+\cdots\right.
$$

In Equation (1), $\gamma$ is the familiar Lorentz factor $1 / \sqrt{1-(\vec{\beta})^{2}}$ with $\vec{\beta} \equiv \vec{v} / c$ with dots $\cdot$ signifying time-derivatives and $\vec{v}$ is the neutrino velocity.

From their neutrino assumption, PSC derived [2] in 2018

$$
\sum_{i} m_{i}<0.12 \mathrm{eV} / \mathrm{c}^{2}
$$

where $i=\mathrm{e}, \mu$ and $\tau$ flavor mass neutrinos.

The KATRIN Consortium [3] is doing a terrestrial laboratory measurement of the electron neutrino mass (from beta-decay) and has ultimate sensitivity (after 5 years data accumulation) of $0.2 \mathrm{eV} / \mathrm{c}^{2}$ for this one neutrino flavor. According to Equation (2), this is a useless effort involving large sums of money, time and human resources, because KATRIN's sensivity is not sufficient and would be expected to return a null result. Thus KATRIN and PSC have a competition, which is the unfolding physics drama. Recently, KATRIN has published their first data review [4] with the result

$$
m_{e}<1.1 \mathrm{eV} / \mathrm{c}^{2}
$$


It has been argued in References $[1,5,6]$ that the neutrino radiation losses, coupled with the expansion of space-time, allowed neutrino condensation to occur. This neutrino condensation would result in Condensed Neutrino Objects (CNO) forming, which would be the Dark Matter everyone is looking for.

Unique signatures have been identified $[5,7]$ that allow observational astronomy to key in on these $\mathrm{CNO}$, which are non-relativistic degenerate objects.

Because of neutrino flavor mixing, we now know that neutrinos have essentially degenerate masses as (e = electron, $\mu=$ muon, $\tau=$ tau) $m_{i=e, \mu, \tau}=m+\Delta m_{i}$ with $\frac{\left|\Delta m_{i}\right|}{m}<1$ and probably $\frac{\left|\Delta m_{i}\right|}{m} \ll 1$. The quantity $m \equiv m_{v}$ is the degenerate neutrino mass (called the neutrino mass scale). In the neutrino equation of state, used for the equation of equilibrium that determines the CNO structure, $m_{v}$ is the parameter.

The CNO mass-radius relationship is predicted to be [5]

$$
M(R) \simeq \frac{1.97462 \times 10^{15} M_{\odot}}{R^{3} m_{v}^{8}},
$$

where $M(R)$ is the mass of the stable CNO having radius $R$ in units of Mpc and $m_{v}$ is in units $\mathrm{eV} / \mathrm{c}^{2}$. Using this mass-radius relationship, observational data from weak lensing galaxy clusters has been fitted $[5,6]$ in Figure 1, which allows the neutrino mass scale $m_{v}$ to be identified

$$
m_{v}=0.827 \pm 0.059 \mathrm{eV} / \mathrm{c}^{2} .
$$

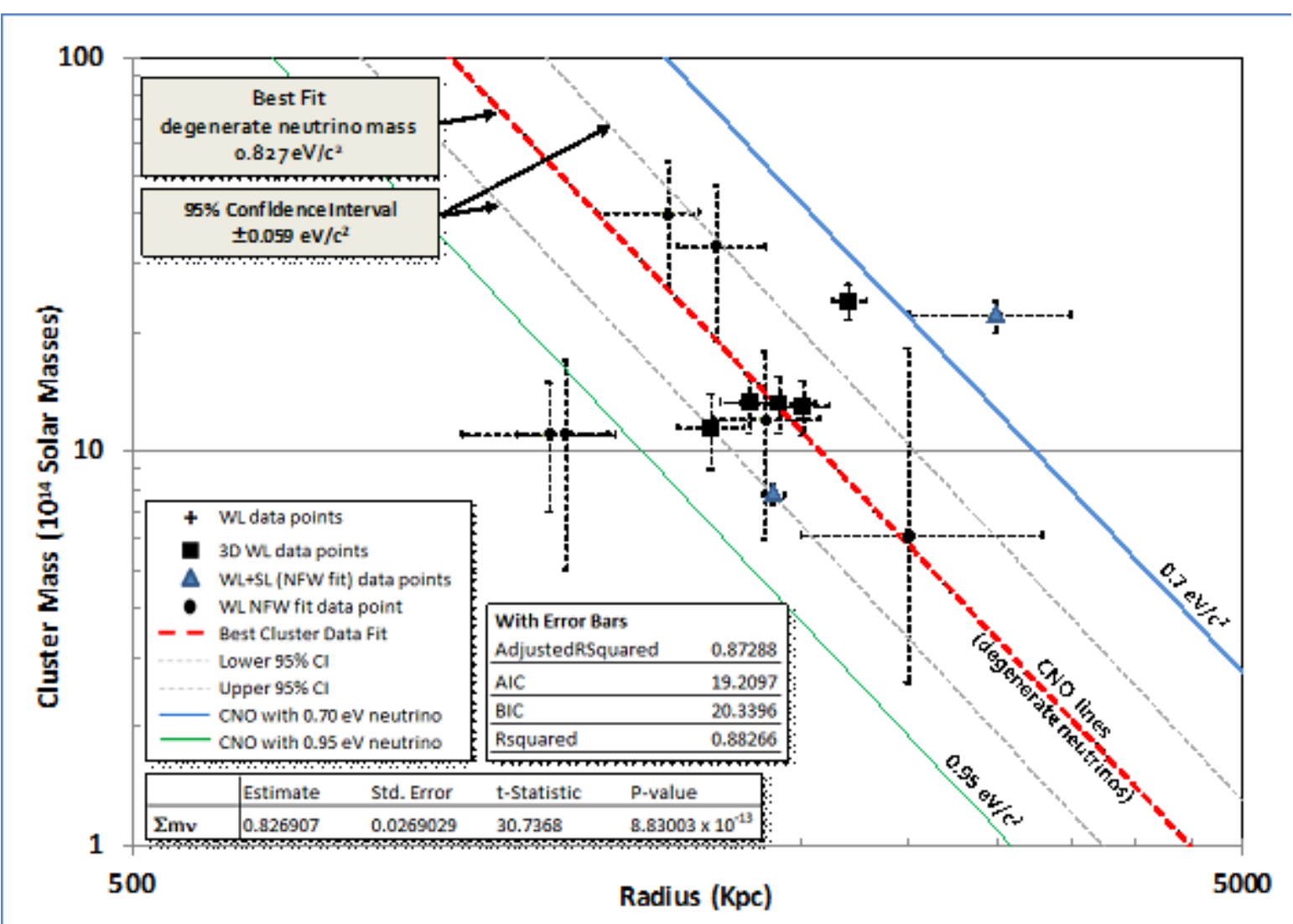

Figure 1. CNO fit to weak lensing data, which gives the neutrino mass scale $[5,6]$.

Thus KATRIN can confirm or refute Equation (5) which will have enormous implications for modern astrophysics and cosmology. Confirmation by KATRIN of a neutrino mass within these bounds would certainly identify CNO theory as the Dark Matter. This finding would suggest that the 
raw Planck satellite data be reprocessed, with now magnetic degrees of freedom replacing the neutrino degrees of freedom, as originally suggested in [5]. Most importantly, the PSC value for their Hubble Constant would be expected to change.

In this paper, in place of weak lensing data with many different galaxy clusters, we instead use a single galaxy cluster's kinematic data (Coma Galaxy Cluster), to derive the neutrino mass scale, $m_{v}$, with $\mathrm{CNO}$ as the Dark Matter.

\section{Using Coma Galaxy Cluster Data to Derive the Neutrino Mass Scale}

It was shown in [8] that matter (cluster gas and member galaxies) embedded within a CNO undergoes simple harmonic motion (SHM), with periods ranging from millions to hundreds of millions of years. The fastest galaxy in a cluster would thus be near the CNO center. Because the Coma Cluster has hundreds of galaxy members, statistically there is a high probability that one would be randomly found near the $\mathrm{CNO}$ center. For this research, we identify the $\mathrm{CNO}$ center as location of the fastest member galaxy. We now derive the fundamental equation that connects the fastest member galaxy speed to the Dark Matter CNO.

The physics of CNO are simple: degeneracy pressure (Pauli Exclusion Principle) supports the Object from gravitational collapse. Being condensed matter, the individual $\mathrm{CNO}$ are distinguished by the value of their Fermi momentum $\left(p_{f}\right)$ at the center. The variable $x=p_{f} / m_{v} c$ (reduced Fermi momentum) enters the equation of equilibrium. The different $\mathrm{CNO}$ are distinguished by their different boundary value at the center $x(0)$. The $\mathrm{CNO}$ that the Local Group seems to be embedded in $[6,7]$ has $x(0)=0.013$ to possible 0.0225 .

\subsection{The Fundamental Equation}

We derive the energy of a galaxy as it starts at an initial radius $r_{\text {int }}$ (formed there) and reaches the center of the CNO. Let $d E_{K E}$ be the (positive) change in kinetic energy that it experiences when it goes a distance $\overrightarrow{d r}$ closer to the CNO center. The work done by the CNO on the infalling galaxy is $d E_{K E}=-\vec{F} \cdot \vec{d} r$, where $\vec{F}=-G M_{C N O}(r) M_{G A L A X Y} / r^{2} \hat{r}$ and $\overrightarrow{d r}=-d r \hat{r}$

$$
\begin{aligned}
d E_{K E} & =-\vec{F} \cdot \overrightarrow{d r} \\
& =-\frac{G M_{\text {cno }}(r) M_{\text {GALAXY }}}{r^{2}} d r
\end{aligned}
$$

Now $d E_{K E}=d\left(\frac{1}{2} M_{G A L A X Y} V^{2}\right)$ So the integration of Equation (6) becomes

$$
V_{\text {final }}^{2}-V_{\text {initial }}^{2}=-\int_{r_{\text {int }}}^{r_{\text {final }}} \frac{2 G M_{\text {cno }}(r)}{r^{2}} d r
$$

Now if we identify that galaxy that has the greatest speed, relative to the Coma Cluster redshift, then that galaxy will have the properties

1. The location of the galaxy is at or near the center of the CNO.

2. The measured speed of that galaxy will satisfy the equation

$$
V_{\text {measured }}^{2}=+\int_{0}^{R_{0}} \frac{2 G M_{\text {cno }}(r)}{r^{2}} d r
$$

where $R_{0}$ is the radius of the $\mathrm{CNO}$ and $M_{\text {cno }}(r)$ is the mass interior to radial coordinate $r$. Equation (8) is the maximum speed a galaxy can have and still be bound by the Dark Matter. Determining $V_{\text {measured }}^{2}$ from the experimental data will allow us to use Equation (8) (evaluated numerically) to determine the $\mathrm{CNO} x(0)$ parameter. 


\subsection{Experimental Data}

Reference [9] gives the list of identified galaxies in the Coma Galaxy Cluster, given by their GMP number [10]. The fastest galaxy belonging to the Coma Cluster is GMP $=3176$, Reference [9], $v_{\text {radial }}=9811 \mathrm{~km} / \mathrm{s}$. With the Coma Cluster red shift corresponding to $6917 \mathrm{~km} / \mathrm{s}$, the relative speed is $2894 \mathrm{~km} / \mathrm{s}$, or squared in $\mathrm{m}^{2} / \mathrm{s}^{2}=8.375 \times 10^{12}$. The CNO configuration (condensed neutrino solutions are explained in Reference [8]) that satisfies this speed squared, using Equation (8), has $x(0)=$ 0.010 , with solution $8.953 \times 10^{12} \mathrm{~m}^{2} / \mathrm{s}^{2}$. Because the data is only line-of-sight speed, then within the uncertainties of the problem, these two quantities are close enough.

Equation (8) only determines the $\mathrm{CNO}$ boundary condition $x(0)$. The Coma Cluster solution $x(0)=0.010$ has mass $\mathcal{M}_{010}$ and radius $\mathcal{R}_{010}$

$$
\begin{gathered}
\mathcal{M}_{010}=\frac{9.809 \times 10^{14}}{m_{v}^{2}} M_{\odot} \\
\mathcal{R}_{010}=\frac{1.2625}{m_{v}^{2}} M p c
\end{gathered}
$$

where $m_{v}$ is in units of $\mathrm{eV} / \mathrm{c}^{2}$. To bound $m_{v}$ we need an estimate of the amount of Dark Matter in the Coma Galaxy Cluster.

The amount of Dark Matter $\mathcal{M}_{\text {Coma }}$ in the Coma Galaxy Cluster has been estimated by reference [11], with $30 \%$ error. Their answer (with the factor 0.85 being their estimate of the Dark Matter contribution):

$$
\mathcal{M}_{\text {Coma }}=.85 \times 1.4 \times 10^{15} h_{70}^{-1} M_{\odot} \equiv Q\left(h_{70}\right)
$$

The variation of $Q$ with $h_{70}$ is

$$
|\delta Q|=Q\left|\frac{\delta h_{70}}{h_{70}}\right|
$$

Noting that the variation of $h_{70}$ in 2019 is $[12,13]$

$$
\frac{\delta h_{70}}{h_{70}} \simeq \pm .1
$$

The maximum Coma Dark Matter max $\mathcal{M}_{\text {Coma }}$ estimated from [11] is

$$
\begin{aligned}
\max \mathcal{M}_{\text {Coma }} & \left.\simeq(1 .+.3)(1+.1) Q\right|_{h_{70}=1}=\left.1.43 Q\right|_{h_{70}=1} \\
& \simeq 1.7017 \times 10^{15} M_{\odot}
\end{aligned}
$$

The minimum Coma Dark Matter min $\mathcal{M}_{\text {Coma }}$ estimated from [11] is

$$
\begin{aligned}
\min \mathcal{M}_{\text {Coma }} & \left.\simeq(1 .-.3)(1-.1) Q\right|_{h_{70}=1}=\left.0.63 Q\right|_{h_{70}=1} \\
& \simeq 0.7497 \times 10^{15} M_{\odot} .
\end{aligned}
$$

Using Equation (9) gives the prediction

$$
0.759 \mathrm{eV} / \mathrm{c}^{2} \lesssim m_{v} \lesssim 1.1 \mathrm{eV} / \mathrm{c}^{2}
$$

Equation (16) is essentially the same as Equation (5), but now derived from kinematic data from a single galaxy cluster, instead of weak lensing data from many clusters of galaxies. We give in Table 1 , the Coma Cluster CNO $x(0)=0.010$ solution. Assuming a Coma Cluster distance of $101.3 \mathrm{Mpc}$ [11], we plot the $\mathrm{CNO}$ in Figure 2. 
Table 1. Dark Matter Condensed Neutrino Objects (CNO) Coma solution.

\begin{tabular}{ccc}
\hline Physical Parameter & Value & Value $\boldsymbol{m}_{\boldsymbol{v}}=\mathbf{0 . 7 5 9} \mathbf{~ \mathbf { V } / \mathbf { c } ^ { \mathbf { 2 } }}$ \\
\hline CNO (Dark Mass) mass & $\frac{9.809 \times 10^{14}}{m_{v}^{2}} \mathrm{M}_{\odot}$ & $1.7 \times 10^{15} \mathrm{M}_{\odot}$ \\
CNO radius & $\frac{1.2625}{m_{v}^{2}} \mathrm{Mpc}$ & $2.191 \mathrm{Mpc}$ \\
CNO center & $(\mathrm{J} 2000) \alpha 125946.28 \delta+274446.1$ & \\
\hline
\end{tabular}

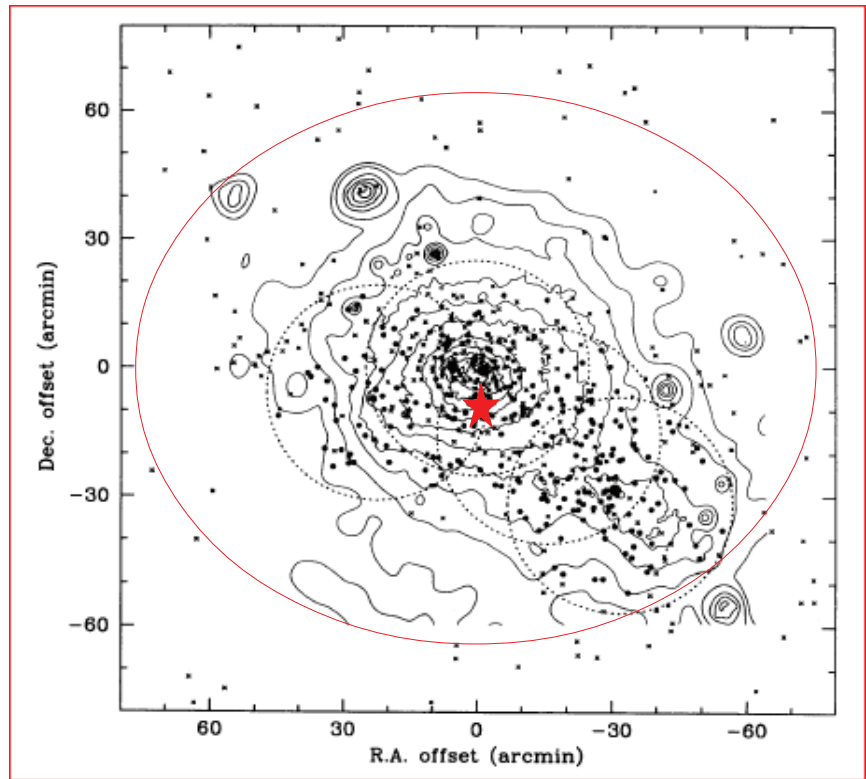

Figure 2. Approximate location of the $\mathrm{CNO}$ center using fastest galaxy GMP $=3176$, which has offsets R.A. +0.0579 arc-minutes and Dec -13.465 arc-minutes. The figure background is taken from reference [9], which shows the actual Coma Galaxy Cluster. At a distance of 101.3 Mpc, the 2.191 Mpc $\mathrm{CNO}$ radius translates to 74.35 arc-minutes.

In Figure 3, we plot the radial variation of the reduced Fermi momentum $x$, while in Figure 4, we plot the Coma Cluster CNO Dark Matter density.

$$
x(0)=0.01
$$

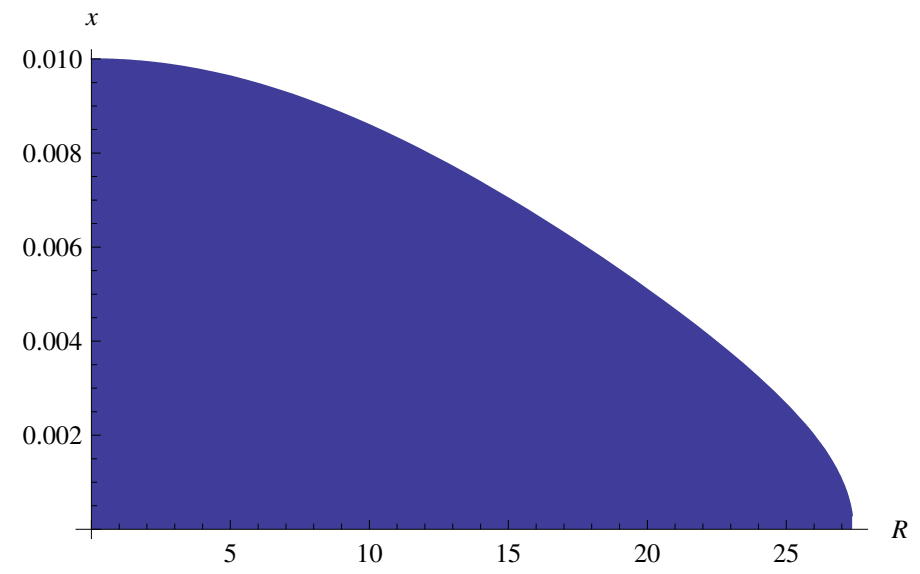

Figure 3. Spatial variation of the reduced Fermi momentum $x$ for the $\mathrm{CNO}$ having boundary value $x(0)=0.010$. The units of length are $46128.98 \mathrm{pc} / m_{v}^{2}$ with $m_{v}$ in units of $\mathrm{eV} / \mathrm{c}^{2}$. 


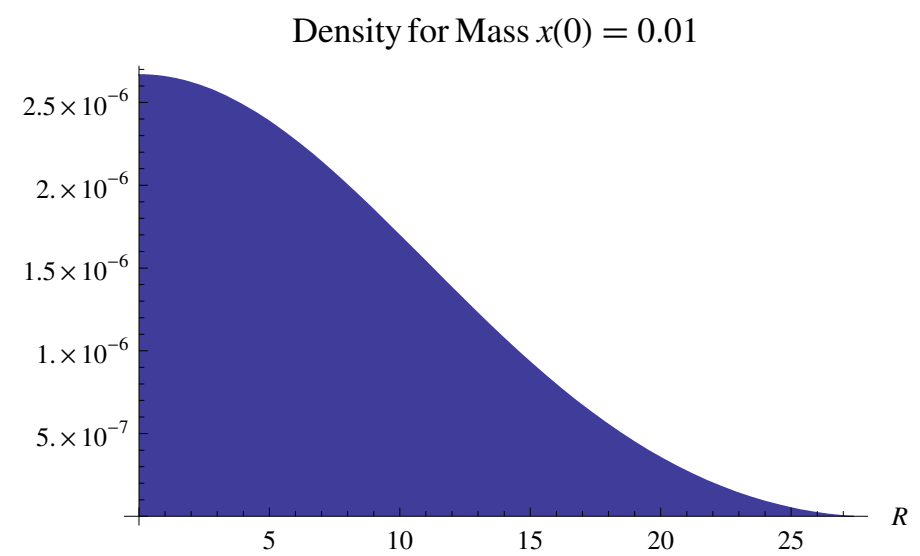

Figure 4. CNO mass density for $x(0)=0.010 \mathrm{CNO}$. The units of mass density are $1.76307 \times 10^{-20} \mathrm{~m}_{v}^{4}$ $\mathrm{gm} / \mathrm{cm}^{3}$ with $m_{v}$ in units of $\mathrm{eV} / \mathrm{c}^{2}$.

\section{Implications of a KATRIN Neutrino Mass Signal}

Assuming a mass signal along the lines of Equation (5):

1. The KATRIN terrestrial experiment will have identified Dark Matter as Condensed Neutrino Objects.

2. The raw Planck satellite microwave data will have to be re-processed with the neutrino degrees of freedom removed, and in their place, magnetic field degrees of freedom inserted.

3. It is expected that the PSC value of the Hubble Constant will be changed.

4. With the advent of $\mathrm{CNO}$ in the Universe, the dynamics of galaxies embedded in $\mathrm{CNO}$ can be studied. That is, the orbits of galaxies embedded in CNO can be computed.

5. The section 26 'Dark Matter' in the Review of Particle Physics [14] which describes Dark Matter as a collection of WIMPS, Axions, etc becomes fiction and the hundreds of references it cites become irrelevant.

6. It is expected that $\mathrm{CNO}$ will greatly influence the evolution of structure in the Early Universe.

7. $\mathrm{CNO}$ have a maximum mass that their internal pressure can support. Exceeding that mass results in CNO gravitational collapse to colossal Black Holes that greatly perturb the Cosmic Microwave Radiation field around them. This provides an experimental signature for their discovery.

Funding: This research received no external funding.

Conflicts of Interest: The author declares no conflict of interest.

\section{References}

1. Morley, P.D.; Buettner, D.J. Instantaneous power radiated from magnetic dipole moments. Astropart. Phys. 2015, 62, 7-11. [CrossRef]

2. Planck Collaboration. Planck 2018 results. VI. Cosmological parameters. arXiv 2019, arXiv1807.06209.

3. KATRIN. Available online: https:/ / www.katrin.kit.edu (accessed on 5 June 2020).

4. KATRIN. An improved upper limit on the neutrino mass from a direct kinematic method by KATRIN. arXiv 2019, arxiv:1909.06048.

5. Morley, P.D.; Buettner, D.J. A dark matter signature for condensed neutrinos. Int. J. Mod. Phys. D 2016, 25, 1650089. [CrossRef]

6. Morley, P.D.; Buettner, D.J. Weak lensing data and condensed neutrino objects. Universe 2017, 3, 81. [CrossRef]

7. Morley, P.D.; Buettner, D.J. Dark matter in the local group of galaxies. Int. J. Mod. Phys. D 2017, $26,1750069$. [CrossRef]

8. Morley, P.D.; Buettner, D.J. SHM of galaxies embedded within condensed neutrino matter. Int. J. Mod. Phys. D 2015, 24, 15500042. [CrossRef] 
9. Colless, M.; Dunn, A.M. Structure and dynamics of the Coma cluster. Astrophys. J. 1996, 458, 435. [CrossRef]

10. Godwin, J.G.; Metcalf, N.; Peach, J.V. Coma cluster 1. A catalogue of magnitudes, colours, ellipticities and position angles for 6724 galaxies in the field of the Coma cluster. Mon. Not. R. Astron. Soc. 1983, 202, 113-124. [CrossRef]

11. Lokas, E.L.; Mamon, G.A. Dark matter distribution in the Coma cluster from galaxy kinematics: Breaking the mass-anisotropy degeneracy. Mon. Not. R. Astron. Soc. 2003, 343, 401. [CrossRef]

12. Freedman, W.L. Cosmology at crossroads: tension with the Hubble constant. arXiv 2017, arxiv:1706.02739.

13. Chen, G.C.; Fassnacht, C.D.; Suyu, S.H.; Rusu, C.E.; Chan, J.H.; Wong, K.C.; Auger, M.W.; Hilbert, S.; Bonvin, V.; Birrer, $\mathrm{S}$; et al. A SHARP view of HoLiCOW: $\mathrm{H}_{0}$ from three time-delay gravitational lens systems with adaptive optics imaging. Mon. Not. R. Astron. Soc. 2019, 490, 1743. [CrossRef]

14. Particle Data Group. Review of particle physics. Phys. Rev. D 2018, 98, 030001. [CrossRef]

(C) 2020 by the author. Licensee MDPI, Basel, Switzerland. This article is an open access article distributed under the terms and conditions of the Creative Commons Attribution (CC BY) license (http:// creativecommons.org/licenses/by/4.0/). 